\title{
Successive AF/DF Relaying in the Cooperative DS-CDMA Uplink: Capacity Analysis and its System Architecture
}

\author{
Li Li, Li Wang, Member, IEEE and Lajos Hanzo, Fellow, IEEE \\ School of ECS, University of Southampton, SO17 1BJ, United Kingdom. \\ Tel: +44-23-8059 3125, Fax: +44-23-8059 4508 \\ Email: $\{115 \mathrm{e} 08$, lw05r, lh $\} @$ ecs.soton.ac.uk, http://www-mobile.ecs.soton.ac.uk
}

\begin{abstract}
A successive relaying aided network (SRAN) is designed for a multi-user spread-spectrum scenario conceived for noncoherent (NC) detection in order to convert the typical $50 \%$ half-duplex relaying-induced throughput loss to a potential user-load reduction of the CDMA system, where the NC allows us to avoid the extra power consumption imposed by channel estimation. We commence by evaluating the noncoherent Discrete-input Continuous-output Memoryless Channel (DCMC) capacity of both the Amplify-and-Forward (AF) based and of the Decode-and-Forward (DF) based SRAN in the DS-CDMA uplink. Whilst NC detection has the added benefit of eliminating both the pilot-overhead and power-hungry channel estimation, it tends to form an error-floor at high Doppler frequencies. We mitigate this problem using multiple-symbol detection, which increases the detection complexity upon extending the detection window. Finally, a relay-aided soft-input soft-output MultipleSymbol Differential Sphere Detection (SISO-MSDSD) CDMA regime is proposed, which significantly reduces the system's complexity without sacrificing its performance.
\end{abstract}

\section{INTRODUCTION}

As a benefit of their spatial diversity, Multiple-Input Multiple-Output techniques [1] are capable of efficiently mitigating the deleterious effects of Rayleigh fading channels. However, achieving transmit diversity in the uplink is impractical due to the limited antenna-separation of shirt-pocket-sized mobiles. Fortunately, the family of cooperation techniques heralded by van der Meulen in [2] is capable of achieving uplink transmit diversity by forming a virtual antenna array (VAA) in a distributed fashion. However, the conventional twophase cooperative system [3] incurs a severe multiplexing loss due to the half-duplex transmit/receive constraint of practical transceivers. A beneficial technique of recovering the halfduplex relaying induced throughput loss was advocated in [4] [5], where the successive relaying regime was proposed and analysed.

We extend the investigation of SRANs from the single-user scenario to a more realistic multi-user scenario of the DSCDMA uplink (UL). The pathloss between the mobile station (MS) and base station (BS) will be mitigated with the aid

The financial support of the European Union under the auspices of the Concerto project, as well as that of the Research Council (RC)-UK under the auspices of the India-UK Advanced Technology Centre known as In-ATC and of the China-UK Science Bridge in 4G Communications is gratefully acknowledged.

Copyright (c) 2012 IEEE. Personal use of this material is permitted However, permission to use this material for any other purposes must be obtained from the IEEE by sending a request to pubs-permissions@ieee.org. of the proposed SRAN, hence the performance of cell-edge users may be remarkably improved [6]. In contrast to [7], the successive relaying induced interferences generated both at the relay node $(\mathrm{RN})$ and at the the destination node (DN) may be eliminated by directly exploiting the classic DS-CDMA principle upon assigning unique, link-specific spreading codes to the potentially interfering links. Naturally, this implies that the orthogonal time-slots used in [5] are replaced by unique, link-specific CDMA spreading codes at the cost of imposing a soft-limit on the number of users that maybe supported, given the limited number of spreading codes.

In order to eliminate the potentially complex channel estimation of coherent detection aided cooperative systems, which consumes extra energy and requires pilots that reduce the overall throughput, the family of noncoherent detection arrangements dispensing with any channel estimation becomes an attractive design alternative. Naturally, they typically impose a $3 \mathrm{~dB}$ performance loss in comparison to their perfect channel estimation based counterparts. However, in practice this penalty is lower than $3 \mathrm{~dB}$, since practical channel estimators may impose a substantial performance loss. Regretfully, however, the noncoherent detector's performance erodes at high normalized Doppler frequencies. As a remedy, the Multiple-Symbol Differential Sphere Detection (MSDSD) algorithm devised by Lampe et al. [8] strikes an attractive trade-off between the BER performance attained and the complexity imposed. Accordingly, a practical noncoherent detector may be conceived. In order to transform the hard decision based MSDSD algorithm to a power-efficient, soft-decisionaided iterative detection scheme, the MSDSD algorithm was further developed to the soft-input soft-output MSDSD (SISOMSDSD) regime in [9].

Against this background, our novel contribution is:

1) We design a SRAN for a multi-user scenario, where a specific interference suppression regime is designed and the effects of both the successive relaying induced interference and of the multiple access interference (MAI) are evaluated.

2) We derive the noncoherent DCMC capacity of both the AF based and of the DF based SRAN in the multi-user scenario, and further exploit the capacity results attained to inform our cooperative-protocol-selection based system design.

3) We specifically devise a relay-aided SISO-MSDSD algorithm, which is capable of combining the different re- 
ceived signal streams at the destination, and incorporate it in the DF based SRAN.

4) We demonstrate that by combing the relay-aided SISOMSDSD decoder with the successive relaying regime, the proposed transceiver significantly reduces the system's complexity while operating close to the system's capacity.

The rest of this paper is organised as follows. Our system model is described in Section III Section III derives the noncoherent DCMC capacities of both the AF based and DF based SRANs. Then in Section IV] we design the proposed transceiver architecture based on the capacity analysis provided in Section $\amalg$ and investigate both the complexity and the robustness of the proposed transceiver. Finally, we conclude in Section V.

\section{System OVERVIEW}

A typical scenario of the cooperative DS-CDMA uplink is portrayed in Figure 11 where the MS $s$ roaming close to the edge of the DS-CDMA cell activates the SRAN regime to improve its communication quality. The activated relays $r_{0}, r_{1}$ and the BS $d$ are specifically labelled in Figure 1

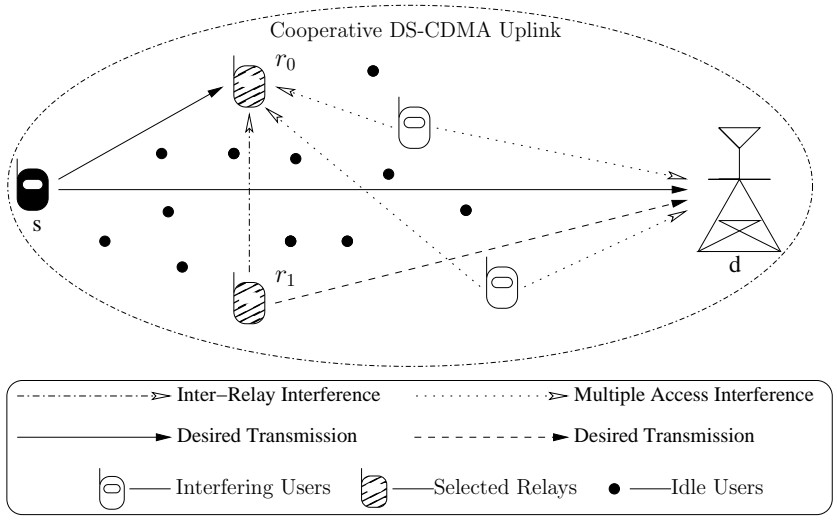

Fig. 1: The successive relaying aided cooperative DS-CDMA uplink topology.

The path-loss gain achieved by the reduced transmission distance experienced in cooperative systems is introduced below. As detailed in [10], the average path-loss gains of the Sourceto-Relay link $\left(S R_{i}\right)$ and Relay-to-Destination link $\left(R_{i} D\right)$ with respect to the Source-to-Destination link (SD) are given by $G_{s r_{i}}=\left(\frac{D_{s d}}{D_{s r_{i}}}\right)^{\alpha}, i=0,1$ and $G_{r_{i} d}=\left(\frac{D_{s d}}{D_{r_{i} d}}\right)^{\alpha}, i=0,1$, respectively, where the notation $D_{a b}, a, b \in\left\{s, r_{0}, r_{1}, d\right\}$ represents the distance between node $a$ and node $b$. Throughout this paper, the path-loss exponent is fixed to $\alpha=3$ for representing a typical urban area.

To simplify our analysis, we assume that the SRAN has a symmetric topology, which implies that $D_{s r_{0}}, D_{r_{0} d}, G_{s r_{0}}$ and $G_{r_{0} d}$ are identical to $D_{s r_{1}}, D_{r_{1} d}, G_{s r_{1}}$ and $G_{r_{1} d}$, respectively. Let us define the transmit power of the MS $s$ for a conventional single-link direct-transmission based DSCDMA uplink as $P_{\text {total }}$. For the sake of a fair comparison between the system employing the proposed SRAN and that operating without a SRAN, we assume that the overall transmit power of the SRAN aided DS-CDMA uplink, which consists of the transmit power $P_{s}$ of MS $s$ and the transmit power $P_{r_{i}}$ of relay $r_{i}$, is also $P_{\text {total }}$. Explicitly, no sophisticated powerallocation scheme is considered here - we simply equally allocate the total power $P_{\text {total }}$ to the source and relay, i.e. we have $P_{s}=P_{r_{i}}=P=1 / 2 P_{\text {total }}$.

Furthermore, all the possible propagation paths between the $s, r_{i}$ and $d$ are assumed to be the correlated time-selective generalized block-fading Rayleigh channels of [11], where the fading coefficients $h_{a b}[k], a, b \in\left\{s, r_{0}, r_{1}, d\right\}$ of the channel between node $a$ and node $b$ fluctuate in a correlated manner over the block period rather than remaining constant, and then switch to another in itself correlated, but independently fading block with respect to the previous block. The independent and identical distribution (i.i.d) assumption from one block to the next [12] is convenient for information-theoretic analysis as it allows us to focus on a single block in studying the capacity. The correlated block-fading period of the channel is represented by $T_{b}$, and the variance $\sigma_{a b}^{2}, a, b \in\left\{s, r_{0}, r_{1}, d\right\}$ of any channel fading coefficient $h_{a b}[k]$ is assumed to be unity.

More details about the SRAN, especially the transmission processes of the different cooperative phases are depicted in Figure 2, where the source transmissions are segmented into identical-length transmission frames of $L$ symbols.

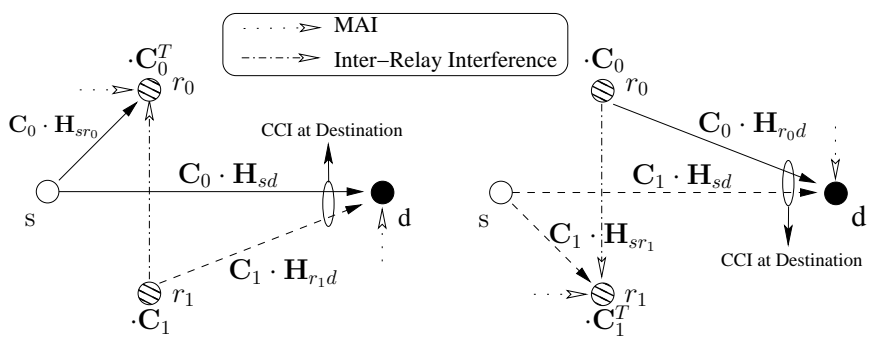

(a) Even Phase " $l \in\{0,2,4, \cdots\}$ "

(b) Odd Phase " $l \in\{1,3,5, \cdots\}$ "

Fig. 2: The successive relaying aided transmission process in its different communication phases.

Observe in Figure 2 that in the even phase $s$ broadcasts its information stream, while $r_{0}$ listens to $s$ and $r_{1}$ forwards the signals received from $s$ during the most recent phase. In the consecutive odd phase, $s$ continues to broadcast its next information stream, but the roles of the relays $r_{0}$ and $r_{1}$ alternate. Similar to [5] [13], we assume that the transmissions of the SRAN depicted in Figure 2 are perfectly aligned to form a synchronous system. As detailed in [5] [13], an impediment of the SRAN is the interference generated among the relays, which is referred to the inter-relay interference (IRI). The IRI is further aggravated by the co-channel interference (CCI) between the signals transmitted from the source node (SN) and relay node $(\mathrm{RN})$, both of which are simultaneously received at the destination node (DN), as seen in Figure 2 As a benefit of combining DS-CDMA with the SRAN philosophy, the inherent ability of CDMA to deal with the multipath effects assists the SRAN in overcoming its interference-limited behaviour [14]. 


\section{A. Concise Introduction of Notations}

Boldface characters are utilized to represent the matrices and vectors. Many notations will be represented in the form of $\mathbf{X}_{a b}^{l}[k]$, which normally means that the physical object $\mathbf{X}_{a b}^{l}[k]$ is associated with the network nodes $a$ and $b$, and occurs in the $k^{t h}$ symbol duration of the $l^{t h}$ frame. A bar over the boldface characters is utilized to emphasize that the matrix (or vector) is constructed from other matrices (or vectors) in a specific manner. The notations in the form of $\mathcal{I}_{a b}^{l}[k]$ represent the interference generated by node $a$ and imposed on node $b$, where the interference is multiplied by a PN sequence. If the multiplication by a PN sequence is omitted as a result of executing the despreading operation, we replace $\mathcal{I}_{a b}^{l}[k]$ with $I_{a b}^{l}[k]$. The tilde in $\tilde{n}_{a}$ is used to emphasize that it is an amplified and faded noise variable.

\section{B. Limitations of Directly Embedding the DS-CDMA Uplink in the SRAN}

In conventional DS-CDMA systems, the SN $s$ employs a single user-specific pseudo-noise (PN) sequence for spreading each individual user's modulated signals. Obverse the successive relaying aided uplink in Figure 2, which portrays the situation that the signal components received from both the $\mathrm{SN}$ and the RN at the DN are spread by the same PN CDMA sequence, albeit this violates the orthogonal codedivision principle of DS-CDMA.

Then, upon considering the AF based SRAN, the transmit power of the RN $r_{i}$ assigned to the $k^{t h}$ symbol of the $(l+$ $1)^{s t}$ frame consists of four components received during the most recent frame: $P_{s}^{l}[k]$ contributed by the SN's broadcast signal, $P_{r_{\bar{i}}}^{l}[k]$ imposed by the IRI, $P_{\mathrm{MAI}}^{l}[k]$ imposed by the MAI and $\sigma^{2}$ representing the AWGN noise, which may be simply formulated by omitting the index $k$ as

$$
\begin{gathered}
P_{r_{i}}^{l+1}=f_{A M_{r_{i}}}^{2}\left\{G_{s r_{i}} P_{s}^{l}+G_{r_{\bar{i}} r_{i}} P_{r_{\bar{i}}}^{l}+P_{\mathrm{MAI}}^{l}+\sigma^{2}\right\} \\
P_{r_{\bar{i}}}^{l}=f_{A M_{r_{\bar{i}}}}^{2}\left\{G_{s r_{\bar{i}}} P_{s}^{l-1}+G_{r_{i} r_{\bar{i}}} P_{r_{i}}^{l-1}+P_{\mathrm{MAI}}^{l-1}+\sigma^{2}\right\} \\
\vdots \\
P_{r_{1}}^{2}=f_{A M_{r_{1}}}^{2}\left\{G_{s r_{1}} P_{s}^{1}+G_{r_{0} r_{1}} P_{r_{0}}^{1}+P_{\mathrm{MAI}}^{1}+\sigma^{2}\right\},
\end{gathered}
$$

where the amplification factor $f_{A M_{r_{i}}}^{2}$ constrains the transmitted power of the RN to $P_{r_{i}}=P$. Naturally, the noise component received by $r_{\bar{i}}$ in the $(l-1)^{s t}$ frame will contaminate the transmitted signal of $r_{i}$ in the $(l+1)^{s t}$ frame, as seen by substituting (2) into (1). This process is then continued recursively. Based on the assumption that $P_{s}^{l}, P_{\mathrm{MAI}}^{l}$ and the Signal-to-Noise-Ratio (SNR) remain constant for the different frames, and proceeding backwards from the $(l+1)^{s t}$ to the $2^{\text {nd }}$ frame, the variance of the total recursively accumulated noise component in $P_{r_{i}}^{l+1}$ may be approximated by

$$
\begin{aligned}
\operatorname{Var}\left[N_{\text {total }}\right] & =\sigma^{2} f^{2}\left[1+f^{2} G+\left(f^{2} G\right)^{2}+\cdots+\left(f^{2} G\right)^{l-1}\right] \\
& \approx \sigma^{2} f^{2} \lim _{l \rightarrow \infty} \sum_{n=0}^{l-1}\left(f^{2} G\right)^{n}=\frac{f^{2} \cdot \sigma^{2}}{1-f^{2} G}
\end{aligned}
$$

where $f$ and $G$ represent $f_{A M_{r_{i}}}$ and $G_{r_{i} r_{i}}$, respectively. This reveals that all the noise generated in different frames at the different relays will be consistently scaled and accumulated during the transmission process of the AF based SRAN. Quantitatively, they impose an extra noise component having a variance of $\frac{f^{2} \cdot \sigma^{2}}{1-f^{2} G}$ on the DN, which cannot be mitigated by the single despreading operation at the $\mathrm{DN} 1$.

\section{The DS-CDMA Based Interference Suppression Regime}

Let $\mathbf{C}_{i}=\left[c_{i}[1], c_{i}[2], \cdots, c_{i}[Q]\right], i=0,1$ denote a pair of PN sequences having a cross-correlation (CCL) of $\gamma_{01}$ and a spreading factor of $Q$. Observe at RN $r_{0}$ of Figure 2 that the received AWGN vector $\mathbf{n}[k]$ of a symbol-duration consists of $Q$ chip-related AWGN samples $n[(k-1) Q+q]$, which may be formulated as [15]: $\mathbf{n}[k]=[n[(k-1) Q+1], n[(k-$ 1) $Q+2], \cdots, n[(k-1) Q+Q]]$, where $n[(k-1) Q+q] \sim$ $\mathcal{C N}\left(0, \sigma^{2} / Q\right)$. After despreading by $\mathbf{C}_{0}^{T}$, the corresponding term of $\mathbf{n}[k]$ is given by

$$
\eta_{1}=\sum_{q_{1}=1}^{Q} n\left[(k-1) Q+q_{1}\right] c_{0}\left[q_{1}\right] \sim \mathcal{C N}\left(0, \sigma^{2}\right),
$$

where $\eta_{1}$ is a Gaussian variable. If $\eta_{1}$ is further spread by $\mathbf{C}_{0}$ and then despread by $\mathbf{C}_{1}^{T}$, we obtain

$$
\eta_{2}=\sum_{q_{2}=1}^{Q} \eta_{1} c_{0}\left[q_{2}\right] c_{1}\left[q_{2}\right] .
$$

Observe that $\eta_{1}$ of (5) obeys a Gaussian distribution in statistical terms, but each of its realizations becomes a specific value in (6). Hence (6) may be rewritten as

$$
\eta_{2}=\eta_{1} \sum_{q_{2}=1}^{Q} c_{0}\left[q_{2}\right] c_{1}\left[q_{2}\right]=\eta_{1} \gamma_{01} .
$$

This implies that the power of the AWGN vector $\mathbf{n}[k]$ cannot be reduced by a single combined spread-despread operation, but it can definitely be mitigated by multiple spread-despread operations.

Based on the above analysis, a specifically arranged DSCDMA regime is designed here in order to circumvent the potential noise accumulation process, and to mitigate both the successive relaying induced interferences and the MAI imposed on the cooperative DS-CDMA uplink. In more detail, at the SN $s$, the modulated symbols $S^{l}[k]$ are alternately spread by $\mathbf{C}_{0}$ and $\mathbf{C}_{1}$ from frame to frame $(l=0,1, \cdots)$. If the $\mathrm{AF}$ protocol is employed, the received signals are firstly despread by $\mathbf{C}_{i}^{T}$ in the listening mode of $\mathrm{RN} r_{i}$, and then directly spread by $\mathbf{C}_{i}$ in the transmit mode of RN $r_{i}$ before the amplification operation. Hence, when we employ an appropriate $\mathrm{PN}$ sequence to suppress the interfering signal transmitted by the RNs (e.g. the IRI) at the receiver (RN or $\mathrm{DN}$ ), according to (7), the AF noise component inherent in the interference will be simultaneously suppressed. This implies

\footnotetext{
${ }^{1}$ For simplifying the analysis of the noise accumulation problem in the AF based SRAN, the path-loss reduction factor $G_{a b}, a, b \in\left\{s, r_{0}, r_{1}, d\right\}$, and the MAI power $P_{M A I}^{l}$, as well as the SNR are assumed to be constant over a number of consecutive frames. The instantaneous channel fading coefficient $h_{a b}[k]$ is also replaced by its variance $\sigma_{a b}^{2}$, which is assumed to be unity. Based on these simplifying assumptions, Eqs. 1) 4 were derived, which are physically plausible from a long-term average perspective.
} 
that the noise accumulation process is indeed avoided. If the DF protocol is employed, the remodulated symbols $\tilde{S}^{l}[k]$ are always spread by $\mathbf{C}_{i}$ in the $\mathrm{RN} r_{i}$. Thus our spreading scheme also guarantees that the two different components of the signal received at the DN, namely those, which correspond to the SN's transmitted signal and to the RN's forwarded signal, respectively, are always spread by different PN sequences. These operations are also illustrated in Figure 2

\section{General Form of the DN's Received Signals}

We assume that the channel's fading coefficients $h_{a b}^{l}[k]$ maintain the same value in different chip-durations of the same symbol. Hence, the $k^{t h}$ received signal of the $l^{t h}$ frame at the DN may be formulated as

$$
\begin{aligned}
\mathbf{y}^{l}[k]= & \sqrt{G_{s d}} h_{s d}^{l}[k] \mathbf{c}_{s}^{l}[k]+\sqrt{G_{r_{i} d}} h_{r_{i} d}^{l}[k] \mathbf{c}_{r_{i}}^{l}[k] \\
& +\mathcal{I}_{s^{M} d}^{l}[k]+\mathbf{n}_{d}^{l}[k],
\end{aligned}
$$

where $i \equiv(l+1) \bmod 2 ; \bar{i} \equiv l \bmod 2 ;$ and $l=0,1, \cdots$. The SN's broadcast signal is given by $\mathbf{c}_{s}^{l}[k]=S^{l}[k] \cdot \mathbf{C}_{\bar{i}}$ and the RN's forwarded signal by $\mathbf{c}_{r_{i}}^{l}[k]=R_{i}^{l}[k] \cdot \mathbf{C}_{i}$. Moreover, the spread AWGN vector $\mathbf{n}_{d}^{l}[k]$ obeys $\mathcal{E}\left\{\mathbf{n}_{d}^{l}{ }^{H}[k] \mathbf{n}_{d}^{l}[k]\right\}=$ $\frac{1}{Q} \sigma^{2} \mathbf{I}_{Q}$, where $\mathbf{I}_{Q}$ denotes the identity matrix having a dimension of $Q$. We assume having $(M+1)$ users, namely the desired user plus $M$ interfering users, and the transmissions of the $M$ interfering users are asynchronous with the transmissions of the desired user. In more detail, the MAI imposed by the $M$ interfering users on the DN is given by $\mathcal{I}_{s^{M} d}^{l}[k]=\sum_{m=1}^{M} \sqrt{G_{s_{m} d}} h_{s_{m} d}^{l}[k] S_{s_{m}}^{l}[k] \cdot \mathbf{C}_{s_{m}}$.

\section{E. AF Based SRAN Model}

When employing the AF protocol, the RN's forwarded signal $\mathbf{c}_{r_{i}}^{l}[k]$ of (8) can be detailed as

$$
\begin{aligned}
\mathbf{c}_{r_{i}}^{l}[k]= & f_{A M_{r_{i}}} \times\left\{\sqrt{G_{s r_{i}}} h_{s r_{i}}^{l-1}[k] \mathbf{c}_{s}^{l-1}[k]\right. \\
& \left.+\mathcal{I}_{r_{\bar{i}} r_{i}}^{l-1}[k]+\mathcal{I}_{s^{M} r_{i}}^{l-1}[k]+\mathbf{n}_{r_{i}}^{l-1}[k]\right\} .
\end{aligned}
$$

The component $\mathcal{I}_{r_{\bar{i}} r_{i}}^{l-1}[k]$ in (9) captures the effect of IRI after the combined despread-spread operation of $\mathrm{RN} r_{i}$, which can be detailed as

$$
\mathcal{I}_{r_{\bar{i}} r_{i}}^{l-1}[k]=\gamma_{\bar{i} i} \sqrt{G_{r_{\bar{i}} r_{i}}} h_{r_{\bar{i}} r_{i}}^{l-1}[k] R_{\bar{i}}^{l-1}[k] \cdot \mathbf{C}_{i},
$$

where the CCL between the PN sequence $\mathrm{C}_{a}$ and the PN sequence $\mathbf{C}_{b}$ is commonly represented by $\gamma_{a b}$. We specifically assign a pair of PN sequences having CCL of $-1 / Q$ as $\mathbf{C}_{\vec{i}}$ and $\mathbf{C}_{i}$ in order to significantly reduce the IRI effects, i.e. we have $\gamma_{\bar{i} i}=-1 / Q$. Then, after the combined despread-spread operation, the MAI imposed on $r_{i}$ during the $(l-1)^{s t}$ frame becomes

$$
\begin{aligned}
\mathcal{I}_{s^{M} r_{i}}^{l-1}[k] & =\tilde{I}_{s^{M} r_{i}}^{l-1}[k] \cdot \mathbf{C}_{i} \\
& =\sum_{m=1}^{M} \gamma_{m i} \sqrt{G_{s_{m} r_{i}}} h_{s_{m} r_{i}}^{l-1}[k] S_{s_{m}}^{l-1}[k] \cdot \mathbf{C}_{i} .
\end{aligned}
$$

From the central limit theorem [16], the summation of $M$ independent random variables can be modelled by the Gaussian distribution. Hence the component $\tilde{I}_{s^{M} r_{i}}^{l-1}[k]$ in (11) may be approximated by a Gaussian variable. Based on the Gaussian approximation method [17], the variance of $\tilde{I}_{s^{M} r_{i}}^{l-1}[k]$ may be approximated by

$$
\operatorname{Var}\left[\tilde{I}_{s^{M} r_{i}}^{l-1}\right] \approx \sum_{m=1}^{M} \frac{1}{3 Q} G_{s_{m} r_{i}} P_{s_{m}}
$$

Regretfully, the power of the MAI imposed on the RN is unknown, but we may utilize the MAI imposed on the DN to approximately model it. Then, based on the assumption that the DS-CDMA system has a perfect power control, we may readily derive the following relationship

$$
\sum_{m=1}^{M} G_{s_{m} d} P_{s_{m}}=M P_{s} ; \sum_{m=1}^{M} G_{s_{m} r_{i}} P_{s_{m}}=\lambda \cdot M P_{s},
$$

where the factor $\lambda$ is determined by the ratio of the MAI imposed on the RN $r_{i}$ to the MAI imposed on the BS $d^{2}$.

By substituting (13) to (12), (12) is rewritten as

$$
\operatorname{Var}\left[\tilde{I}_{s^{M} r_{i}}^{l-1}\right] \approx \frac{\lambda}{3 Q} M P_{s}
$$

In order to constrain the transmitted power of the RN $r_{i}$ to $P_{r_{i}}$ [18], and invoking (10) as well as (14), the amplification factor of $r_{i}$ is represented by $f_{A M_{r_{i}}}=\sqrt{\frac{P_{r_{i}}}{G_{s r_{i}} P_{s}+\frac{1}{Q^{2}} G_{r_{\bar{i}} r_{i}} P_{r_{\bar{i}}}+\frac{\lambda}{3 Q} M P_{s}+\sigma^{2}}}$. We note that $\mathbf{c}_{r_{i}}^{l}[k]$ of (9) corresponds to the $k^{t h}$ modulated source symbol of the previous frame, namely to $S^{l-1}[k]$, instead of corresponding to $S^{l}[k]$ transmitted by the SN in the present frame. This particular property of the SRAN was also described in [13, (Fig.1d)].

At the DN, the different components of the received signal $\mathbf{y}^{l}[k]$ can be resolved by appropriately configuring the chipwaveform matched-filter for the different spreading codes. When the filter is matched to the waveform $\mathbf{C}_{\bar{i}}$, the signal directly transmitted by the SN will contribute the main component of the despread signal, while the RN's forwarded signal and the interfering users' signals become the interference. Hence, the associated output of the chip-waveform matchedfilter is given by

$$
z_{s}^{l}[k]=\sqrt{G_{s d}} h_{s d}^{l}[k] S^{l}[k]+I_{r_{i} d}^{l}[k]+I_{s^{M} d}^{l}[k]+n_{d}^{l}[k],
$$

where $I_{r_{i} d}^{l}[k]$ accounts for the relay's forwarded signal component after the despreading operation. Recalling the analysis of Section [I-C according to (7), the noise term $\mathbf{n}_{r_{i}}^{l-1}[k]$ in (9) will be suppressed along with the other components of (9) by the CCL $\gamma_{i i}$. Then $I_{s^{M} d}^{l}[k]$ is the despread version of $\mathcal{I}_{s^{M} d}^{l}[k]$ in (8), whose variance may be calculated by a process similar to (11), (14). Hence the variances of the interference terms in (15) are obtained as $\operatorname{Var}\left[I_{r_{i} d}^{l}\right]=\frac{1}{Q^{2}} G_{r_{i} d} P_{r_{i}} ; \operatorname{Var}\left[I_{s^{M} d}^{l}\right] \approx$ $\frac{1}{3 Q} M P_{s}$. Moreover, we have $n_{d}^{l}[k] \sim \mathcal{C N}\left(0, \sigma^{2}\right)$

Similarly, the despread signal extracted from $\mathbf{y}^{l}[k]$ but dominated by the RN's forwarded signal $\mathbf{c}_{r_{i}}^{l}[k]$ can be expressed

\footnotetext{
${ }^{2}$ The actual value of $\lambda$ will vary owing to different network topologies. As one possible value, 2.0 is assigned to $\lambda$ in our simulations.
} 
as

$$
\begin{aligned}
z_{r_{i}}^{l}[k]= & f_{A M_{r_{i}}} \sqrt{G_{s r_{i}}} \sqrt{G_{r_{i} d}} h_{s r_{i}}^{l-1}[k] h_{r_{i} d}^{l}[k] S^{l-1}[k] \\
& +I_{r_{\bar{i}} r_{i}}^{l-1}[k]+I_{s^{M} r_{i}}^{l-1}[k]+I_{s d}^{l}[k]+I_{s^{M} d}^{l}[k] \\
& +\tilde{n}_{r_{i}}^{l-1}[k]+n_{d}^{l}[k],
\end{aligned}
$$

where $I_{r_{i} r_{i}}^{l-1}[k], I_{s^{M} r_{i}}^{l-1}[k]$ and $\tilde{n}_{r_{i}}^{l-1}[k]$ are the amplified and faded, then despread versions of $\mathcal{I}_{r_{\bar{i}} r_{i}}^{l-1}[k], \mathcal{I}_{s^{M} r_{i}}^{l-1}[k]$ and $\mathbf{n}_{r_{i}}^{l-1}[k]$ in (9). $I_{s d}^{l}[k]$ is the interference imposed by the SN's broadcast signal $\mathbf{c}_{s}^{l}[k]$. Their variances are summarized as: $\operatorname{Var}\left[I_{r_{\bar{i}} r_{i}}^{l-1}\right]=\left(\frac{1}{Q^{2}} G_{r_{\bar{i}} r_{i}} P_{r_{i}}\right) f_{A M_{r_{i}}}^{2} G_{r_{i} d} ; \operatorname{Var}\left[I_{s^{M} r_{i}}^{l-1}\right] \approx$ $\left(\frac{\lambda}{3 Q} M P_{s}\right) f_{A M_{r_{i}}}^{2} G_{r_{i} d} ; \operatorname{Var}\left[I_{s d}^{l}\right]=\frac{1}{Q^{2}} G_{s d} P_{s} ; \operatorname{Var}\left[\tilde{n}_{r_{i}}^{l-1}\right]=$ $\left(\sigma^{2}\right) f_{A M_{r_{i}}}^{2} G_{r_{i} d}$.

\section{F. DF Based SRAN Model}

When employing the DF protocol, the despread signal of the $(l-1)^{s t}$ frame at $r_{i}$ associated with $S^{l-1}[k]$ is given by

$$
\begin{aligned}
& y_{r_{i}}^{l-1}[k]=\sqrt{G_{s r_{i}}} h_{s r_{i}}^{l-1}[k] S^{l-1}[k]+\gamma_{\bar{i} i} \sqrt{G_{r_{\bar{i}} r_{i}}} h_{r_{\bar{i}} r_{i}}^{l-1}[k] R_{\bar{i}}^{l-1}[k] \\
& +\sum_{m=1}^{M} \gamma_{m i} \sqrt{G_{s_{m} r_{i}}} h_{s_{m} r_{i}}^{l-1}[k] S_{s_{m}}^{l-1}[k]+n_{r_{i}}^{l-1}[k],
\end{aligned}
$$

which is similar to 9. In order to avoid the typical errorpropagation problem of the DF protocol, we ensure that the signals at RN $r_{i}$ are forwarded only when correctly decoded. Consequently, the signal forwarded by $r_{i}$ is formulated as

$$
\mathbf{c}_{r_{i}}^{l}[k]=\tilde{S}^{l}[k] \cdot \mathbf{C}_{i}, \quad \tilde{S}^{l}[k]= \begin{cases}S^{l-1}[k] & \text { perfect detection } \\ 0 & \text { else. }\end{cases}
$$

At the DN, when the chip-waveform matched-filter is applied to the waveform of $\mathbf{C}_{\bar{i}}$, the resultant output corresponding to the information bearing symbol $S^{l}[k]$ is given by

$$
z_{s}^{l}[k]=\sqrt{G_{s d}} h_{s d}^{l}[k] S^{l}[k]+I_{r_{i} d}^{l}[k]+I_{s^{M} d}^{l}[k]+n_{d}^{l}[k],
$$

where the definitions of the terms of $I_{r_{i} d}^{l}[k], I_{s^{M} d}^{l}[k]$ and $n_{d}^{l}[k]$ are similar to that in (15), and their variances are also equivalent to that in (15). Similarly, another despread signal extracted from $\mathbf{y}^{l}[k]$ of $(8)$ but corresponding to the information-bearing symbol $S^{l-1}[k]$ may be formulated as

$$
z_{r_{i}}^{l}[k]=\sqrt{G_{r_{i} d}} h_{r_{i} d}^{l}[k] \tilde{S}^{l-1}[k]+I_{s d}^{l}[k]+I_{s^{M} d}^{l}[k]+n_{d}^{l}[k] .
$$

\section{NONCOHERENT DCMC CAPACITY OF THE Successive Relaying Aided Network}

Both the AF and the DF protocols may be invoked by the SRAN. Hence we have to find the appropriate cooperation protocol for a given transmission scenario. The optimum cooperation-protocol-selection can be achieved with the aid of capacity analysis. As mentioned above, noncoherent detection will be employed in order to avoid the complexity as well as pilot overhead of channel estimation. Hence we focus on deriving the noncoherent DCMC capacity of both the AF based and DF based SRAN. Then in Section IV we will exploit this in order to inform and motivate our design.

The transmission arrangement of the twin-relay-aided successive relaying procedure may be viewed as the superimposed transmissions of two half-duplex three-terminal cooperative networks [13] conditioned by that the CCI and IRI imposed on the SRAN have been equivalently induced in the two half-duplex three-terminal cooperative networks. This is also illustrated in Figure 2, where the transmissions represented by the solid lines in the even phase and odd phase constitute one of the half-duplex three-terminal cooperative networks, namely Coop-I. Similarly, the transmissions represented by the dashed lines in the even phase and odd phase constitute another one, namely Coop-II. Hence we split the DCMC capacity derivation process into two steps. First we attain the noncoherent DCMC capacity of the half-duplex three-terminal cooperative network and then extend the result obtained in the first step to the SRAN. Furthermore, as stated in Section III based on the i.i.d assumption of the channel, in this capacity analysis section, we can focus our attention on a single transmission block, which is randomly extracted from a transmission frame and happen to experience a complete fading block.

\section{A. Noncoherent DCMC Capacity for the AF Based SRAN in the Cooperative DS-CDMA Uplink}

According to above philosophy, the noncoherent DCMC capacity of the AF based SRAN is constituted by the sum of the capacities of the AF based sub-networks Coop-I and Coop-II, which is formulated as

$$
C_{\text {Successive }}^{\mathrm{AF}}=C_{\mathrm{Coop}-\mathrm{I}}^{\mathrm{AF}}+C_{\mathrm{Coop}-\mathrm{II}}^{\mathrm{AF}} \cdot
$$

In the sub-network Coop-I, during the even phase broadcast interval of length $T_{s}$, a block of the modulated source symbols $\mathbf{S}^{l}=\left[S^{l}[k+1], S^{l}[k+2], \cdots, S^{l}\left[k+T_{b}\right]\right]^{T}$ is broadcast by the SN. The transmissions via the SD link generate the signal vector $\mathbf{z}_{s}^{l}=\left[z_{s}^{l}[k+1], z_{s}^{l}[k+2], \cdots z_{s}^{l}\left[k+T_{b}\right]\right]^{T}$, which is actually constituted by the associated despread signals received at the DN, as detailed in (15). According to [16] again, the interference components in (15) are treated as a Gaussian variable. Consequently, the overall equivalent noise component imposed on the vector $\mathbf{z}_{s}^{l}$ may be expressed as

$$
\mathbf{n}_{s}^{l}=\left[\begin{array}{c}
I_{r_{1} d}^{l}[k+1]+I_{s^{M} d}^{l}[k+1]+n_{d}^{l}[k+1] \\
\vdots \\
I_{r_{1} d}^{l}\left[k+T_{b}\right]+I_{s^{M} d}^{l}\left[k+T_{b}\right]+n_{d}^{l}\left[k+T_{b}\right]
\end{array}\right] .
$$

Furthermore, the associated fading coefficients of the SD link are represented by $\mathbf{h}_{s d}=\left[h_{s d}^{l}[k+1], h_{s d}^{l}[k+2], \cdots, h_{s d}^{l}[k+\right.$ $\left.\left.T_{b}\right]\right]^{T}$. Hence, given the definitions $\sqrt{3} \boldsymbol{\Phi}_{s d}=P \mathcal{E}\left\{\mathbf{h}_{s d} \mathbf{h}_{s d}{ }^{H}\right\}$ and $\overline{\mathbf{S}}=\operatorname{diag}\left\{\mathbf{S}^{l}\right\}$, the covariance matrix $\boldsymbol{\Psi}_{s d}$ of the despread received signal vector $\mathbf{z}_{s}^{l}$ is given by

$$
\begin{aligned}
\mathbf{\Psi}_{s d} & =\mathcal{E}\left\{\mathbf{z}_{s}^{l} \mathbf{z}_{s}^{l} \mid \mathbf{S}^{l}\right\} \\
& =\overline{\mathbf{S}} \Phi_{s d} \overline{\mathbf{S}}^{H}+\left\{\left[\frac{G_{r_{1} d}}{Q^{2}}+\frac{M}{3 Q}\right] P+\sigma^{2}\right\} \mathbf{I}_{T_{b}},
\end{aligned}
$$

where both the successive relaying induced interference and the MAI are included in the evaluation. The detailed derivation of (23) is not provided here owing to space limitations, but similar procedures may be found in [8] and [19].

\footnotetext{
${ }^{3}$ From now on, the assumption that $P_{s}=P_{r_{i}}=P$ is utilized in order to
} simplify the form of the equations. 
Similarly, the signal vector $\mathbf{z}_{r_{0}}^{l+1}=\left[z_{r_{0}}^{l+1}[k+1], z_{r_{0}}^{l+1}[k+\right.$ $\left.2], \cdots z_{r_{0}}^{l+1}\left[k+T_{b}\right]\right]^{T}$ represents another replica of the same modulated source symbol block $\mathbf{S}^{l}$, which is generated by the consecutive transmissions from the $\mathrm{SN}$ to the $\mathrm{RN}$ during the broadcast phase (i.e. even phase) of duration $T_{s}$, and then to the DN during the multiple-access phase (i.e. odd phase) of duration $T_{r}$. The equivalent fading coefficients of the associated channels are represented by $\mathbf{h}_{s r_{0} d}=\left[h_{s r_{0}}^{l}[k+1]\right.$. $\left.h_{r_{0} d}^{l+1}[k+1], \cdots, h_{s r_{0}}^{l}\left[k+T_{b}\right] \cdot h_{r_{0} d}^{l+1}\left[k+T_{b}\right]\right]^{T}$. Then the overall equivalent noise component $\mathbf{n}_{r_{0}}^{l+1}$ imposed on the vector $\mathbf{z}_{r_{0}}^{l+1}$ is composed by all the interference and noise components in (16). The relevant covariance matrix is formulated as

$$
\begin{aligned}
& \Psi_{\mathbf{n}_{r_{0}}^{l+1}}=\left\{\frac{G_{r_{0} d}\left\{\left[\frac{G_{r_{1} r_{0}}}{Q^{2}}+\frac{\lambda M}{3 Q}\right] P+\sigma^{2}\right\} P}{\left[G_{s r_{0}}+\frac{G_{r_{1} r_{0}}}{Q^{2}}+\frac{\lambda M}{3 Q}\right] P+\sigma^{2}}\right. \\
&\left.+\left[\frac{G_{s d}}{Q^{2}}+\frac{M}{3 Q}\right] P+\sigma^{2}\right\} \times \mathbf{I}_{T_{b}} .
\end{aligned}
$$

Similar to our previous analysis, given the definition $\boldsymbol{\Phi}_{s r_{0} d}=P f_{A M_{r_{0}}}^{2} G_{s r_{0}} G_{r_{0} d} \mathcal{E}\left\{\mathbf{h}_{s r_{0} d} \mathbf{h}_{s r_{0} d}^{H}\right\}$, the covariance matrix $\Psi_{s r_{0} d}$ of the signal vector $\mathbf{z}_{r_{0}}^{l+1}$ may be formulated as

$$
\boldsymbol{\Psi}_{s r_{0} d}=\overline{\mathbf{S}} \boldsymbol{\Phi}_{s r_{0} d} \overline{\mathbf{S}}^{H}+\boldsymbol{\Psi}_{\mathbf{n}_{r_{0}}^{l+1}} .
$$

In the capacity analysis, a single-input-multiple-output (SIMO) system having $N_{T}=1$ transmit antenna, and $N_{R}=2$ receive antennas may be employed to equivalently model the AF based sub-network Coop-I, where the transmissions via the SD link during the broadcast phase of Coop-I can be viewed as the transmissions from the single transmit antenna to one of the two receive antennas in the equivalent SIMO system. Similarly, the consecutive transmissions from the SN to the $\mathrm{RN}$ ensuing during the broadcast phase, and then to the DN during the multiple-access phase in Coop-I can be treated as the transmissions from the single transmit antenna to another receive antenna, while experiencing the equivalent channel's fading effect and incurring the same interference-plus-noise impairment in the SIMO system. Hence the entire input-output relation of the equivalent SIMO system may be formulated by

$$
\overline{\mathbf{Z}}=\overline{\mathbf{S}} \cdot \overline{\mathbf{H}}+\overline{\mathbf{N}},
$$

where we have the definitions $\overline{\mathbf{Z}}=\left[\begin{array}{ll}\mathbf{z}_{s}^{l} & \mathbf{z}_{r_{0}}^{l+1}\end{array}\right], \overline{\mathbf{H}}=$ $\left[\begin{array}{ll}\mathbf{h}_{s d} & \mathbf{h}_{s r_{0} d}\end{array}\right]$ and $\overline{\mathbf{N}}=\left[\begin{array}{ll}\mathbf{n}_{s}^{l} & \mathbf{n}_{r_{0}}^{l+1}\end{array}\right]$

It was pointed out by Ding et al. [20] that the channel's fading coefficients experienced by $\mathbf{z}_{r_{0}}^{l+1}$, namely by the relayed versions of the source codewords, involve a product of Gaussian random variables, which is no longer Gaussian. Nevertheless, fortunately, Ding et al. further demonstrated that the ergodic channel capacity of the AF aided single-relay system based on the Gaussian assumption is still achievable, provided that the covariance matrix of the system's input signal is diagonal, and the diagonal elements may be obtained by solving the resultant optimization problems invoking multidimensional integrals. In the spirit of [20], we may approximate the capacity of the equivalent SIMO system based on the Gaussian assumption. Hence, according to [21], the probability density function (pdf) of $\overline{\mathbf{Z}}$ conditioned on $\overline{\mathbf{S}}$ is readily expressed as

$$
\operatorname{Pr}(\overline{\mathbf{Z}} \mid \overline{\mathbf{S}})=\frac{1}{\left[\pi^{T_{b} N_{T}} \operatorname{det}(\mathbf{\Psi})\right]^{N_{R}}} \cdot \exp \left[-\operatorname{tr}\left(\overline{\mathbf{Z}}^{H} \boldsymbol{\Psi}^{-1} \overline{\mathbf{Z}}\right)\right]
$$

where the conditional covariance matrix $\boldsymbol{\Psi}$ is calculated by

$$
\begin{aligned}
\boldsymbol{\Psi}= & \overline{\mathbf{S}}\left(\boldsymbol{\Phi}_{s d}+\boldsymbol{\Phi}_{s r_{0} d}\right) \overline{\mathbf{S}}^{H} \\
& +\boldsymbol{\Psi}_{\mathbf{n}_{r_{0}}^{l+1}}+\left\{\left[\frac{G_{r_{1} d}}{Q^{2}}+\frac{M}{3 Q}\right] P+\sigma^{2}\right\} \mathbf{I}_{T_{b}} .
\end{aligned}
$$

Let us define $\beta_{1}=\frac{T_{s}}{T_{s}+T_{r}}$ and $\beta_{2}=\frac{T_{r}}{T_{s}+T_{r}}$, which was introduced for evaluating the effect of the source- and relay-transmission duration ratio of a conventional singlerelay aided two-phase cooperative network, as detailed in [22]. Apparently, the AF based sub-network Coop-I has the inherent relationship of $\beta_{1}=\beta_{2}=0.5$, which can be hence represented by a single variable of $\beta$. Then, according to [23] and [24], the capacity of Coop-I may be readily formulated as

$$
C_{\mathrm{Coop-I}}^{\mathrm{AF}}=\frac{\beta}{T_{b}} I(\overline{\mathbf{S}} ; \overline{\mathbf{Z}})=\frac{\beta}{T_{b}}[H(\overline{\mathbf{Z}})-H(\overline{\mathbf{Z}} \mid \overline{\mathbf{S}})],
$$

where the conditional entropy $H(\overline{\mathbf{Z}} \mid \overline{\mathbf{S}})$ can be calculated in a closed form as

$$
\begin{aligned}
H(\overline{\mathbf{Z}} \mid \overline{\mathbf{S}}) & =-\int \operatorname{Pr}(\overline{\mathbf{Z}}, \overline{\mathbf{S}}) \ln [\operatorname{Pr}(\overline{\mathbf{Z}} \mid \overline{\mathbf{S}})] \mathrm{d} \overline{\mathbf{Z}} \mathrm{d} \overline{\mathbf{S}} \\
& =\log _{2}\left\{\mathrm{e}^{T_{b}} \cdot[\operatorname{det}(\pi \mathbf{\Psi})]^{N_{R}}\right\} .
\end{aligned}
$$

However, the entropy $H(\overline{\mathbf{Z}})$ cannot be evaluated in a closed form. A practical approach to its numerical evaluation is constituted by the following Monte-Carlo integration

$$
H(\overline{\mathbf{Z}})=-\mathcal{E}\left\{\log _{2}\left[\frac{1}{M_{c}^{T_{b}}} \sum_{\overline{\mathbf{S}}^{\prime} \in \chi} \frac{\exp \left[-\operatorname{tr}\left(\overline{\mathbf{Z}}^{H} \mathbf{\Psi}^{-1} \overline{\mathbf{Z}}\right)\right]}{[\operatorname{det}(\pi \mathbf{\Psi})]^{N_{R}}}\right]\right\} .
$$

Since each element of the $T_{b}$-component differentially encoded signal vector $\mathbf{S}^{l}$ is chosen independently from an $M_{c}$-point constellation set $\mathcal{M}_{c}$ with equal probabilities, the number of all hypothetically transmitted symbol matrices $\overline{\mathbf{S}}^{\prime}$ equals to $M_{c}^{T_{b}}$ and the notation $\chi$ in 31 represents the set of all hypothetical values of $\overline{\mathbf{S}}^{\prime}$. Additionally, in line with [25], we also define the so-called equivalent transmit SNR, which is determined by the ratio of the transmit power with respect to the receiver's noise. This definition is convenient, but unconventional, since in contrast to the classic SNR, these two quantities are measured at physically different points. Now, we are ready to calculate the value of $C_{\mathrm{Coop-I}}^{\mathrm{AF}}$. Then, we can simply attain the capacity of the sub-network Coop-II following a similar process. According to (21), the noncoherent DCMC capacity of the AF based SRAN becomes available.

\section{B. Noncoherent DCMC Capacity for the DF Based SRAN in the Cooperative DS-CDMA Uplink}

Similarly to (21), the noncoherent DCMC capacity of the DF based SRAN is constituted by the sum of the capacities 
of the DF based sub-networks Coop-I and Coop-II, which is formulated as follows

$$
C_{\text {Successive }}^{\mathrm{DF}}=C_{\text {Coop-I }}^{\mathrm{DF}}+C_{\mathrm{Coop}-\mathrm{II}}^{\mathrm{DF}} \cdot
$$

In the broadcast interval of sub-network CoopI, presented by solid lines in the even phase of Figure 2, the source signal block $\mathbf{S}^{l}$ is broadcast, which is received and further despread to the signal blocks $\quad \mathbf{y}_{r_{0}}^{l}=\left[y_{r_{0}}^{l}[k+1], y_{r_{0}}^{l}[k+2], \cdots, y_{r_{0}}^{l}\left[k+T_{b}\right]\right]^{T}$ associated with (17) and $\mathbf{z}_{s}^{l}$ associated with (19), at RN $r_{0}$ and DN, respectively. Then, during the consecutive multiple-access interval, presented by the solid line in the odd phase, the hypothetical source signal block $\tilde{\mathbf{S}}^{l+1}=\left[\tilde{S}^{l+1}[k+1], \tilde{S}^{l+1}[k+2], \cdots, \tilde{S}^{l+1}\left[k+T_{b}\right]\right]^{T}$ generated by $\mathrm{RN} r_{0}$ is received at the $\mathrm{DN}$, which is despread to signal block $\mathbf{z}_{r_{0}}^{l+1}$ associated with (20). Hence, based on the upper and lower bounds on the capacity of a half-duplex relaying system provided in [22] and on the fact that the SN remains silent during the odd phase, we can obtain the upper and lower bounds for sub-network Coop-I as

$$
\begin{aligned}
C_{\mathrm{Coop}-\mathrm{I}}^{\mathrm{DF}} \leq \min \{ & \beta_{1} I\left(\mathbf{S}^{l} ; \mathbf{z}_{s}^{l}, \mathbf{y}_{r_{0}}^{l}\right), \\
& \left.\beta_{1} I\left(\mathbf{S}^{l} ; \mathbf{z}_{s}^{l}\right)+\beta_{2} I\left(\tilde{\mathbf{S}}^{l+1} ; \mathbf{z}_{r_{0}}^{l+1}\right)\right\}, \\
C_{\mathrm{Coop}-\mathrm{I}}^{\mathrm{DF}} \geq \min \{ & \beta_{1} I\left(\mathbf{S}^{l} ; \mathbf{y}_{r_{0}}^{l}\right), \\
& \left.\beta_{1} I\left(\mathbf{S}^{l} ; \mathbf{z}_{s}^{l}\right)+\beta_{2} I\left(\tilde{\mathbf{S}}^{l+1} ; \mathbf{z}_{r_{0}}^{l+1}\right)\right\} .
\end{aligned}
$$

Furthermore, in order for the $\mathrm{RN} r_{0}$ to detect the received signal correctly and thus to avoid the potential error propagation problem, the actual source transmission rate, namely $R_{\text {Coop-I }}^{\mathrm{DF}}$, cannot exceed the noncoherent DCMC capacity of the $S R_{0}$ link. Hence we obtain another constraint on the error-freerelaying-based capacity as

$$
R_{\text {Coop-I }}^{\mathrm{DF}} \leq \beta_{1} I\left(\mathbf{S}^{l} ; \mathbf{y}_{r_{0}}^{l}\right)
$$

Accordingly, based on (33), 34) and (35), the error-freerelaying-based noncoherent DCMC capacity of sub-network Coop-I is given by

$$
\begin{aligned}
C_{\text {Coop-Even }}^{\mathrm{DF}}=\min \{ & \beta_{1} I\left(\mathbf{S}^{l} ; \mathbf{y}_{r_{0}}^{l}\right), \\
& \left.\beta_{1} I\left(\mathbf{S}^{l} ; \mathbf{z}_{s}^{l}\right)+\beta_{2} I\left(\tilde{\mathbf{S}}^{l+1} ; \mathbf{z}_{r_{0}}^{l+1}\right)\right\} .
\end{aligned}
$$

The covariance matrices associated with $I\left(\mathbf{S}^{l} ; \mathbf{y}_{r_{0}}^{l}\right)$, $I\left(\mathbf{S}^{l} ; \mathbf{z}_{s}^{l}\right)$ and $I\left(\tilde{\mathbf{S}}^{l+1} ; \mathbf{z}_{r_{0}}^{l+1}\right)$ are represented by $\mathbf{\Psi}_{s r_{0}}, \mathbf{\Psi}_{s d}$ and $\Psi_{r_{0} d}$, respectively. Since the covariance matrix of the despread signals is the crucial element engaged in evaluating the noncoherent DCMC capacity, we formulate them explicitly as

$$
\begin{aligned}
\boldsymbol{\Psi}_{s r_{0}}= & P G_{s r_{0}} \overline{\mathbf{S}} \mathcal{E}\left\{\mathbf{h}_{s r_{0}} \mathbf{h}_{s r_{0}}^{H}\right\} \overline{\mathbf{S}}^{H} \\
& +\left\{\left[\frac{G_{r_{1} r_{0}}}{Q^{2}}+\frac{\lambda M}{3 Q}\right] P+\sigma^{2}\right\} \mathbf{I}_{T_{b}} \\
\mathbf{\Psi}_{s d}= & P G_{s d} \overline{\mathbf{S}} \mathcal{E}\left\{\mathbf{h}_{s d} \mathbf{h}_{s d}^{H}\right\} \overline{\mathbf{S}}^{H} \\
& +\left\{\left[\frac{G_{r_{1} d}}{Q^{2}}+\frac{M}{3 Q}\right] P+\sigma^{2}\right\} \mathbf{I}_{T_{b}} \\
\mathbf{\Psi}_{r_{0} d}= & P G_{r_{0} d} \overline{\mathbf{S}} \mathcal{E}\left\{\mathbf{h}_{r_{0} d} \mathbf{h}_{r_{0} d}^{H}\right\} \overline{\mathbf{S}}^{H} \\
& +\left\{\left[\frac{G_{s d}}{Q^{2}}+\frac{M}{3 Q}\right] P+\sigma^{2}\right\} \mathbf{I}_{T_{b}},
\end{aligned}
$$

where the interference components are treated as noise contaminating the desired signal having the equivalent power. The $\mathbf{h}_{s r_{0}}$ and $\mathbf{h}_{r_{0} d}$ denote the fading coefficient vectors corresponding to $S R_{0}$ link and $R_{0} D$ link, respectively.

After obtaining the relevant covariance matrices, the noncoherent DCMC capacity of sub-network Coop-I can be attained by implementing a similar process according to 29 ,, 30 ) and (31). According to the symmetry between sub-network CoopI and Coop-II, we may argue that the noncoherent DCMC capacity solution of sub-network Coop-II is also available. Hence the evaluation of the noncoherent DCMC capacity of the DF based SRAN is deemed completed. Moreover, no code-rate-optimization was considered here for the sake of simplifying the analysis. Hence we equally split the time between the broadcast and cooperation phases, which directly leads to $\beta_{1}=\beta_{2}=0.5$, when calculating (36).

\section{Simulation Results and Analysis}

Let us define $\theta=\frac{D_{s r_{i}}}{D_{r_{i}} d}$, and assume that $D_{r_{0} r_{1}}$ equals to the relatively smaller value between $D_{s r_{i}}$ and $D_{r_{i} d}$. Based on the symmetrical structure of the proposed SRAN assumed in Section [I] the exact proportions of $D_{s r_{i}}, D_{r_{i} d}, D_{s d}$ and $D_{r_{0} r_{1}}$ associated with different $\theta$ values are summarized in Table II where the distance $D_{s d}$ is always normalized to unity. Hence the process that the value of $\theta$ increases represents the fact that the selected relays roam further and further away from $\mathrm{SN}$, but closer and closer to DN. Employing the normalized

\begin{tabular}{|c|cccc|}
\hline$\theta=\frac{D_{s r_{i}}}{D_{r_{i} d}}$ & $D_{s r_{i}}$ & $D_{r_{i} d}$ & $D_{s d}$ & $D_{r_{0} r_{1}}$ \\
\hline$\frac{1}{3}$ & 0.262 & 0.785 & 1.0 & 0.262 \\
\hline$\frac{1}{2}$ & 0.357 & 0.714 & 1.0 & 0.357 \\
\hline 1.0 & 0.577 & 0.577 & 1.0 & 0.577 \\
\hline 2.0 & 0.714 & 0.357 & 1.0 & 0.357 \\
\hline 3.0 & 0.785 & 0.262 & 1.0 & 0.262 \\
\hline
\end{tabular}

TABLE I: Distance proportions with respect to $\theta$.

distance list of Table II the path-loss gain associated with a certain $\theta$ may be obtained according to [10]. Furthermore, in our simulations, the Gold sequence set having a spreading factor of 127 is employed.

A cooperative-user-selection scheme is employed, where the effect of the RN's position is considered. The corresponding simulation results of $C_{\text {Successive }}^{\mathrm{AF}}$ as evaluated from (21), (29), (30) as well as (31) are displayed in Figure 3 , 


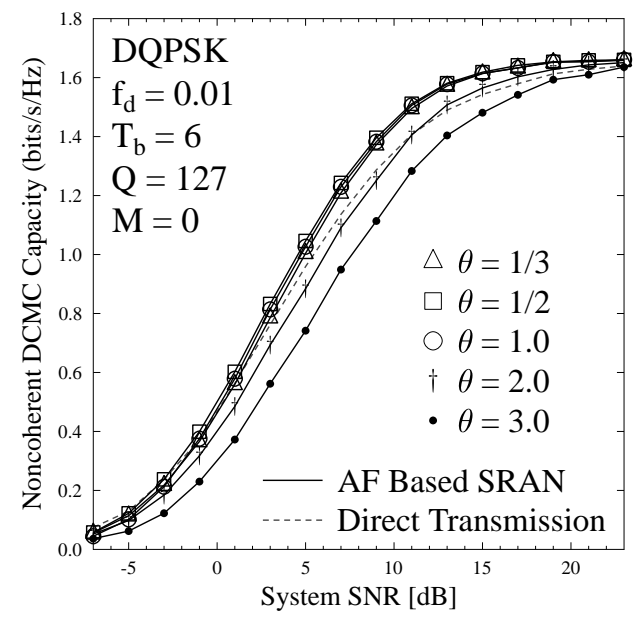

Fig. 3: The effect of the geographic position of the $\mathrm{RN}$ on

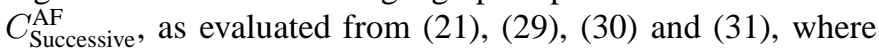
a normalized Doppler frequency of $f_{d}=0.01$ is assumed.

where the following $\mathrm{RN}$ positions expressed in terms of $\theta \in\left\{\frac{1}{3}, \frac{1}{2}, 1.0,2.0,3.0\right\}$ were considered and the number of interfering users was fixed to $M=0$.

Observe in Figure 3 that the capacity of the SN's uplink employing the AF based SRAN exceeds that of the conventional single-link direct transmission structure, when appointing RNs sufficiently close to the SN. By contrast, it results in a degraded capacity compared to the conventional direct transmission structure, when appointing RNs close to the DN. In our particular case, appointing the RNs at the position of $\theta=\frac{1}{2}$ is seen to be the best strategy in Figure 3, which slightly improves the capacity $C_{\text {Successive }}^{\mathrm{AF}}$ compared to the scenarios of $\theta=\frac{1}{3}$ and $\theta=1.0$. In our next investigation we fixed the position of RNs to $\theta=\frac{1}{2}$ and focussed our attention on the detrimental effects of having an increased number of interfering users $M$. Specifically, the values of $M \in\{0,32,64\}$ are considered for modelling the scenarios of zero, moderate and heavy MAI, respectively.

The relevant simulation results displayed in Figure 4/demonstrate that the capacity of the AF based SRAN always exceeds that of the direct transmission system, regardless of the number of interfering users. It is more intriguing to find that the capacity advantage of the AF based SRAN increases with respect to the conventional direct transmission system, when the MAI becomes stronger. This implies that in contrast to the conventional DS-CDMA uplink, the cooperative DS-CDMA uplink will exhibit more substantial advantages in high-load situations. Furthermore, it is expected that if we adjust the amplification factor $f_{A M_{r_{i}}}$ with the aid of accurate powercontrol instead of equally splitting the power between the SN and $\mathrm{RN}$, the AF based capacity may be further improved, as also reported in [20], [26]. This benefit may indeed be anticipated, since having a reduced $\mathrm{SN}$ and $\mathrm{RN}$ transmit power results in reduced MAI in comparison to the direct transmission scenario.

Similarly, when the DF based SRAN is employed, the attainable capacity $C_{\text {Successive }}^{\mathrm{DF}}$ associated with different RN positions is characterized in Figure 5 This demonstrates that

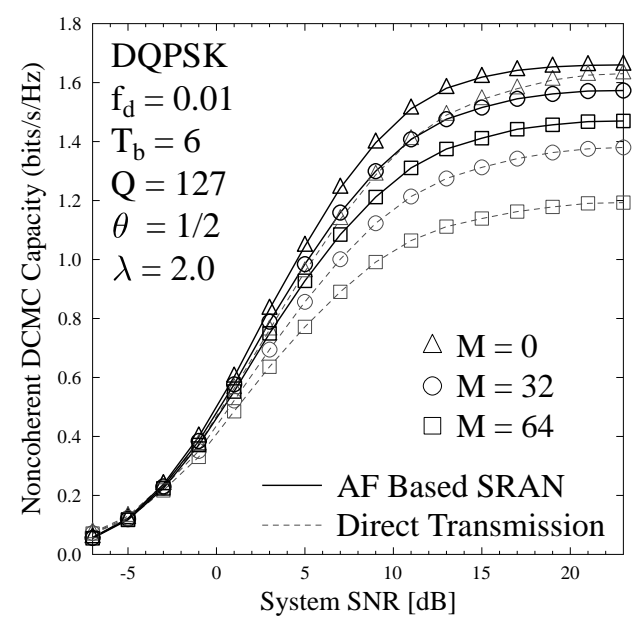

Fig. 4: The noncoherent DCMC capacity $C_{\text {Successive }}^{\mathrm{AF}}$ in zero, moderate and heavy MAI scenarios, as evaluated from (21), (29), (30) and (31), where a normalized Doppler frequency of $f_{d}=0.01$ is assumed.

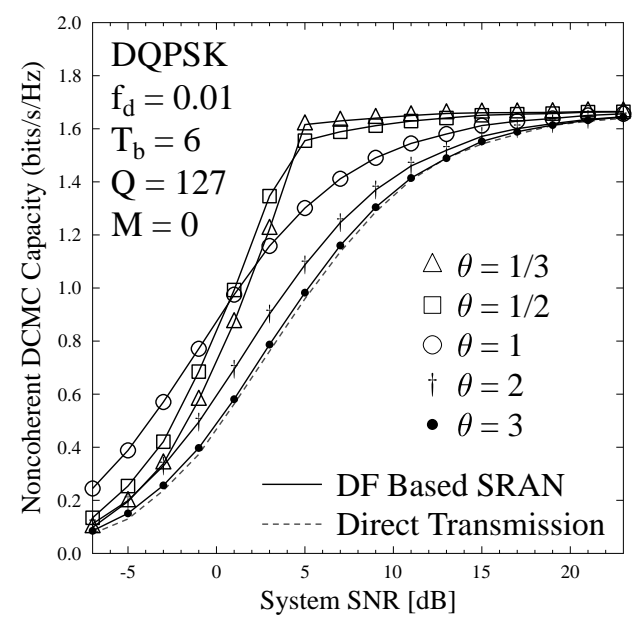

Fig. 5: The effect of the geographic position of the RN on $C_{\text {Successive, }}^{\mathrm{DF}}$ as evaluated from (32), (36) and (37).

appointing RNs, which roam closer to the SN - rather than to the DN - in the DF based SRAN provides a higher capacity. To expound a little further, in the low SNR region $(<1 \mathrm{~dB})$, the DF based system appointing RNs at the position of $\theta=1.0$ achieves the highest capacity. The previously noted phenomenon, namely that the capacity gain achieved by replacing the direct transmission structure with the SRAN increases upon increasing the number of interfering users remains valid, when considering the DF based SRAN, as demonstrated by Figure 6

Upon comparing Figure 3 to Figure 5, We observe that the DF based SRAN appointing appropriate RNs outperforms AF based SRAN, especially at low SNRs, i.e. in the lowthroughput region. In this treatise, we configure our SRAN to operate at low SNRs for the sake of saving precious transmit power. Accordingly, instead of the AF scheme, the DF scheme is proposed for our SRAN, where an improved BER performance is expected. 


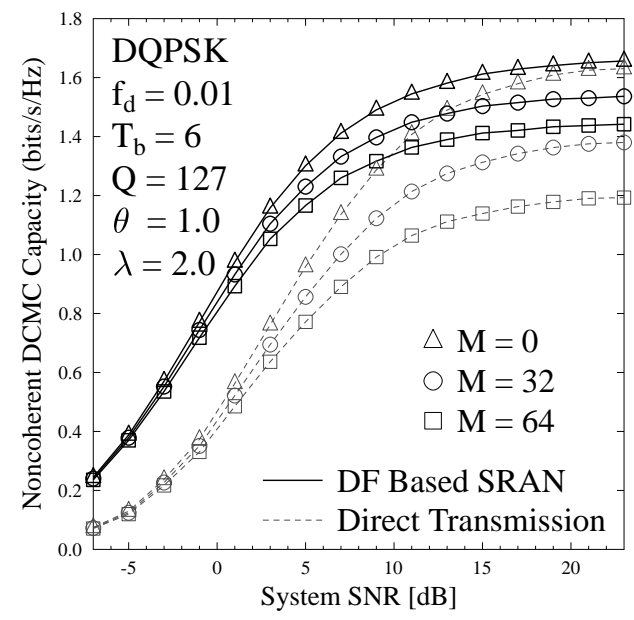

Fig. 6: The noncoherent DCMC capacity $C_{\text {Successive }}^{\mathrm{DF}}$ in zero, moderate and heavy MAI scenarios, as evaluated from (32), (36) and (37).

\section{RELAY-AIDED SISO-MSDSD ASSISTED ITERATIVE DECODING FOR SUCCESSIVE RELAYING}

Based on the analysis provided in Section III] we simply assume that two relays are selected, which employ DF relaying and roam close to the mid-way position between the $\mathrm{SN}$ and DN, where we have $D_{s r_{i}}=D_{r_{i} d}$. In this system design part, a low MAI scenario is assumed for the sake of concentrating on the specific properties of SRANs. Furthermore, we consider a more realistic scenario in this section, where all the channels are assumed to be conventional narrowband correlated timeselective Rayleigh fading channels.

The transceiver architecture specifically designed for our DF based SRAN is portrayed in Figure 7 At the SN, we use a conventional differentially encoded modulator, such as DQPSK depicted at the top right corner of Figure 7 which is further combined with a unity-rate-code's (URC) encoder in order to create a two-stage inner code 4 . Furthermore, a conventional half-rate RSC is employed as the outer code. Hence a three-stage RSC-URC-DM source encoder is created. The corresponding URC decoder assisted three-stage receiver proposed for the relay is also portrayed in Fig.7 In more detail, the RN's receiver consists of three stages, namely the conventional single-path SISO-MSDSD [9] based soft decoder, the URC decoder and the RSC decoder. The extrinsic information and a priori information, represented by $E(\cdot)$ and $A(\cdot)$ respectively, are interleaved and iteratively exchanged within the two-stage inner decoder $I_{\text {inner }}^{r}$ times, before the result is further exchanged between the inner and outer decoders $I_{\text {outer }}^{r}$ times. The motivation of employing this three-stage concatenated decoder architecture is to improve the

\footnotetext{
${ }^{4}$ The URC model has an infinite impulse response (IIR) due to its recursive encoder structure, consequently the EXIT curve of the URC aided inner decoder is capable of approaching the point of perfect convergence at $(1.0,1.0)$ in the EXIT chart, which is a necessary condition for near-capacity operation [6], [27], and for eliminating the potential error floor phenomenon. Therefore, the receiver of the RN is capable of near-perfectly detecting the information bits $\hat{u}_{1}$ bore in the signals received from the SN, at as low SNR values as possible.
}

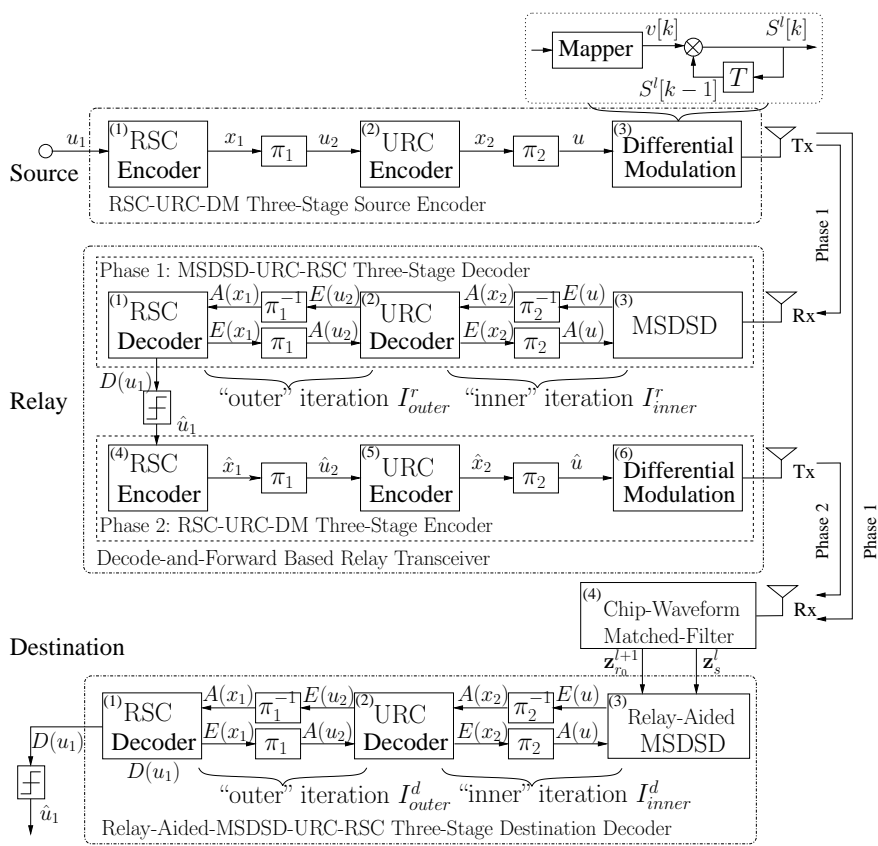

Fig. 7: Schematic of the proposed transceiver in our DF based SRAN.

convergence behavior of the iterative decoder with the aid of the URC decoder, as detailed in [6], [27]. As a benefit, the error propagation problem of the DF scheme is avoided. The RN's transmitter is designed to be identical to the three-stage RSC-URC-DM encoder of the SN. Furthermore, the proposed SISO-MSDSD decoder is also employed at the DN, where we have to ensure that its multiple input signal streams are appropriately time-aligned, so that they correspond to the same differentially modulated symbols. Hence, if the estimates $\hat{u}_{1}$ are correctly generated by the RN's receiver, the differentially modulated symbols produced by the RN's transmitter will be the same as $S^{l}[k]$.

Before introducing the DN's receiver design, we first analyse the proposed SISO-MSDSD algorithm. Initially, the SISO-MSDSD algorithm advocated by Pauli et al. in [9] was invoked for iterative decoding in the direct transmission system, which was then further developed for our cooperative network. The resultant modified SISO-MSDSD algorithm is consequently termed as the relay-aided SISO-MSDSD. This was achieved by developing a sophisticated sphere detection scheme, which is capable of simultaneously dealing with multiple soft-input information streams, where the streams are associated with the same modulated symbols. Instead of processing a single received signal stream, our relay-aided SISO-MSDSD evaluates the a posteriori LLR of the $\mu^{\text {th }}$ bit $u[\mu]$ by simultaneously processing $\Omega$ received signal streams represented by $\left\{\mathbf{z}_{\lambda}\right\}_{\lambda=1,2, \ldots, \Omega}$ as follows

$$
L_{u}[\mu]=\ln \frac{\operatorname{Pr}\left(u[\mu]=b \mid\left\{\mathbf{z}_{\lambda}\right\}_{\lambda=1,2, \ldots, \Omega}\right)}{\operatorname{Pr}\left(u[\mu]=\bar{b} \mid\left\{\mathbf{z}_{\lambda}\right\}_{\lambda=1,2, \ldots, \Omega}\right)}, \quad b \in\{0,1\},
$$

where $\bar{b}$ is the complement of $b$. The resultant relay-aided SISO-MSDSD decoder is employed as the first stage of the overall iterative receiver at the $\mathrm{DN}$, which is then further 
amalgamated with the URC decoder in order to form a twostage inner decoder for appropriately complementing the SN's and RN's transmitter architecture. As described in Section III the despread signal streams of $\mathbf{z}_{s}^{l}$ and $\mathbf{z}_{r_{0}}^{l+1}$ generated at the DN correspond to the same SN's transmitted signal stream $\mathbf{S}^{l}$. Upon substituting them into (38) as $\left\{\mathbf{z}_{\lambda}\right\}_{\lambda=1,2}$, (38) can be rewritten with the aid of Bayes' theorem as

$$
\begin{aligned}
L_{u}[\mu] & =\ln \frac{\operatorname{Pr}\left(u[\mu]=b \mid \mathbf{z}_{s}^{l}, \mathbf{z}_{r_{0}}^{l+1}\right)}{\operatorname{Pr}\left(u[\mu]=\bar{b} \mid \mathbf{z}_{s}^{l}, \mathbf{z}_{r_{0}}^{l+1}\right)} \\
& =\ln \frac{\sum_{\mathbf{v} \in \chi_{: u[\mu]=b}} \operatorname{Pr}\left(\mathbf{z}_{s}^{l} \mid \mathbf{v}\right) \operatorname{Pr}\left(\mathbf{z}_{r_{0}}^{l+1} \mid \mathbf{v}\right) \operatorname{Pr}(\mathbf{v})}{\sum_{\mathbf{v} \in \chi_{: u[\mu]=\bar{b}}} \operatorname{Pr}\left(\mathbf{z}_{s}^{l} \mid \mathbf{v}\right) \operatorname{Pr}\left(\mathbf{z}_{r_{0}}^{l+1} \mid \mathbf{v}\right) \operatorname{Pr}(\mathbf{v})},
\end{aligned}
$$

where the vector $\mathbf{v}$ consists of $\left(T_{b}-1\right)$ QPSK symbols. The relationship of the symbol-vector $\mathbf{v}$ and $\mathbf{S}^{l}$ becomes explicit in the model seen at the top-right corner of Fig 7 Furthermore, $\chi_{: u[\mu]=b}$ represents the set of $\frac{M_{c}^{T_{b}-1}}{2}$ number of legitimate transmitted vectors $\mathbf{v}$, whose $\mu^{t h}$ bit is constrained to $u[\mu]=b$, and similarly, $\chi_{: u[\mu]=\bar{b}}$ is defined as the set corresponding to $u[\mu]=\bar{b}$. Based on (39) and invoking the "sum-max" approximation as well as replacing $\mathbf{S}^{l}$ with a simple notation $\mathbf{s}$, the a posteriori LLR of $u[\mu]$ is further approximated by

$$
\begin{aligned}
& L_{u}[\mu] \\
& \approx \ln \frac{\max _{\mathbf{v} \in \chi_{: u[\mu]=b}} \exp \left\{-\left\|\mathbf{U}^{s} \mathbf{s}\right\|^{2}-\left\|\mathbf{U}^{r_{0}} \mathbf{s}\right\|^{2}+\ln \operatorname{Pr}(\mathbf{v})\right\}}{\max _{\mathbf{v} \in \chi_{: u[\mu]=\bar{b}}} \exp \left\{-\left\|\mathbf{U}^{s} \mathbf{s}\right\|^{2}-\left\|\mathbf{U}^{r_{0}} \mathbf{s}\right\|^{2}+\ln \operatorname{Pr}(\mathbf{v})\right\}} \\
& =-\left\|\mathbf{U}^{s} \hat{\mathbf{s}}_{\mathrm{MAP}}^{b}\right\|^{2}-\left\|\mathbf{U}^{r_{0}} \hat{\mathbf{s}}_{\mathrm{MAP}}^{b}\right\|^{2}+\ln \operatorname{Pr}\left(\hat{\mathbf{v}}_{\mathrm{MAP}}^{b}\right) \\
& \quad+\left\|\mathbf{U}^{s} \hat{\mathbf{s}}_{\mathrm{MAP}}^{\bar{b}}\right\|^{2}+\left\|\mathbf{U}^{r_{0}} \hat{\mathbf{s}}_{\mathrm{MAP}}^{\bar{b}}\right\|^{2}+\ln \operatorname{Pr}\left(\hat{\mathbf{v}}_{\mathrm{MAP}}^{\bar{b}}\right)
\end{aligned}
$$

where $\mathbf{U}^{s}$ can be attained by $\mathbf{U}^{s} \triangleq\left(\mathbf{F} \operatorname{diag}\left\{\mathbf{z}_{s}^{l}\right\}\right)^{*}$, with $\mathbf{F}$ being an upper-triangular matrix obtained by the Cholesky factorization of the matrix $\Psi_{s d}$ of (37). Explicitly, $\mathbf{U}^{r_{0}}$ can be calculated by a similar method, where $\mathbf{F}$ is given this time by the Cholesky factorization of the matrix $\boldsymbol{\Psi}_{r_{0} d}$ of (37). Still referring to (40), $\hat{\mathbf{s}}_{\mathrm{MAP}}^{b}$ is one of the legitimate differentially encoded DQPSK symbol vectors $\mathbf{s}$, which maximizes the term $\left\{-\left\|\mathbf{U}^{s} \mathbf{s}\right\|^{2}-\left\|\mathbf{U}^{r_{0}} \mathbf{s}\right\|^{2}+\ln \operatorname{Pr}(\mathbf{v})\right\}$ involved in the numerator of (40). The signal vector $\hat{\mathrm{s}}_{\mathrm{MAP}}^{b}$ can be attained by implementing a specific sphere detection (SD) algorithm, which is similar to that described in [28]. The SD's search space is constrained to $\chi_{: u[\mu]=b}$. Then $\hat{\mathbf{v}}_{\text {MAP }}^{b}$ is the corresponding QPSK symbol vector, which is uniquely identified by $\hat{\mathbf{s}}_{\mathrm{MAP}}^{b}$. Similarly, if the SD's search space is fixed to $\chi_{: u[\mu]=\bar{b}}, \hat{\mathbf{s}}_{\mathrm{MAP}}^{b}$ and $\hat{\mathbf{v}}_{\mathrm{MAP}}^{\bar{b}}$ are readily obtained.

Our complexity comparison between the single-path SISOMSDSD decoder advocated in [9] and the proposed relayaided SISO-MSDSD decoder is provided in Fig 8 In the spirit of [9], the average number of real-valued multiplication operations (RMO) required for generating a single soft-output during the SISO-MSDSD detection once per iteration is employed here as our complexity measure. If a correlated blockfading period of $T_{b}=6$, and a normalized Doppler frequency of $f_{d}=0.01$ are assumed, the SNR values of $6.05 \mathrm{~dB}$ and $2.12 \mathrm{~dB}$ represent the corresponding "turbo-cliff" points for the single-path SISO-MSDSD assisted system and for the relayaided SISO-MSDSD assisted system, respectively. We will return to these issues later in the context of Fig $9 \mathrm{~b}$. For the sake of a fair comparison, we ensured that both the conventional single-path SISO-MSDSD decoder and the relay-aided SISOMSDSD decoder operated near their associated "turbo-cliff" points. Then we varied the a priori mutual information of the two different SISO-MSDSD decoders and recorded the associated number of RMO required for producing a single soft-output once per iteration. Observe in Fig 8 that the proposed relay-aided SISO-MSDSD decoder approximately doubles the complexity compared to the single-path SISOMSDSD decoder, which is valid right across the entire $a$ priori mutual information region considered. The remaining

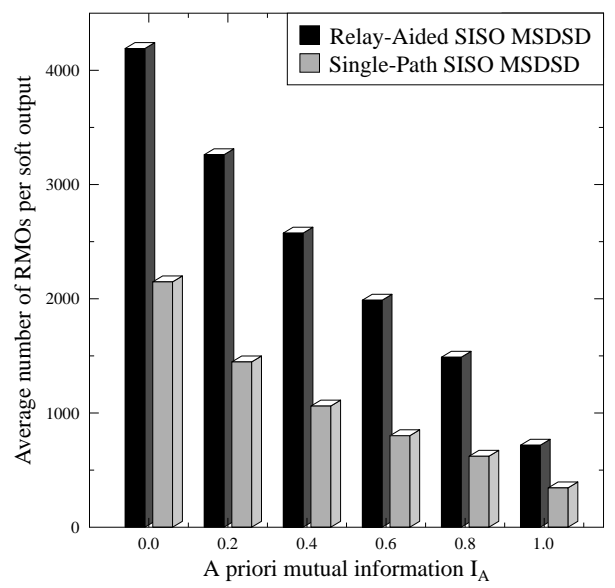

Fig. 8: Complexity comparison between the relay-aided SISOMSDSD decoder and the conventional single-path SISOMSDSD decoder at different a priori mutual information values.

components of the DN's receiver are similar to those of the $\mathrm{RN}$, hence they affect the overall complexity in a similar way.

When designing an iterative decoding aided cooperative system, the distributed turbo coding scheme advocated in [25] is attractive, since it benefits from the iterative information exchange between the direct and relayed versions of the same codeword, which experience uncorrelated fading. Naturally, the improved system performance is achieved at the cost of increasing the complexity imposed by employing an extra iteration stage.

By contrast, the proposed relay-aided SISO-MSDSD algorithm constitutes a realistic method of maintaining a low complexity, where the combination of the information provided by the direct and relayed signal streams, namely by $\mathbf{z}_{s}^{l}$ and $\mathbf{z}_{r_{0}}^{l+1}$ is achieved without an extra iteration stage. More explicitly, in a cooperative network, where the distributed turbo coding principle is employed by invoking the singlepath SISO-MSDSD algorithm, as in [12], each input signal stream is first individually processed by a single-path SISOMSDSD aided turbo decoder within the inner iterative stage of [12, (Figure 5)]. Then the resultant information is passed on to the outer iterative stage, and typically at least two iterations are carried out in order to exchange information between the different input signal streams. Hence, based on our complexity comparisons shown in Fig 8 , it is reasonable to argue that our three-stage relay-aided-MSDSD-URC-RSC decoder is capable 


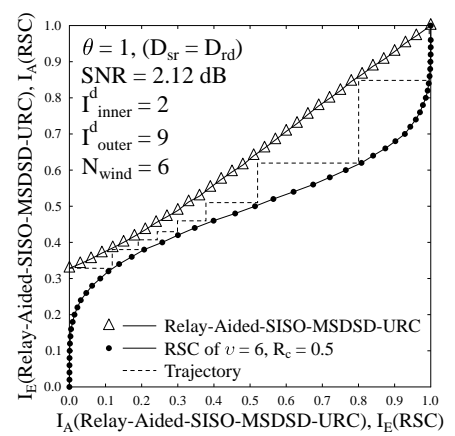

(a) EXIT Chart

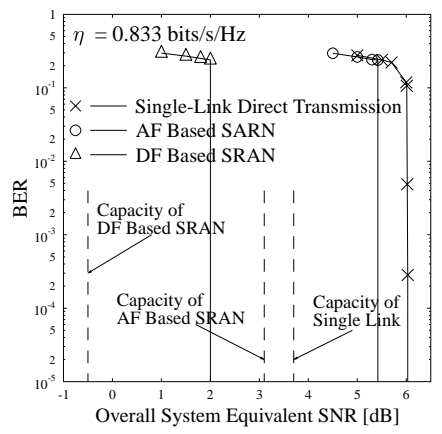

(b) BER performance
Fig. 9: The EXIT characteristic of our proposed destination receiver and the BER performances corresponding to different systems.

of halving the system complexity imposed by the conventional single-path SISO-MSDSD aided distributed turbo decoder. Let

\begin{tabular}{|l|r|}
\hline Channel Model & Time-Selective Block-Fading Channel \\
\hline Path-Loss Exponent & $\alpha=3$ \\
\hline Correlated Fading Block Length & $T_{b}=6$ \\
\hline Normalized Doppler Frequency & $f_{d}=0.01$ \\
\hline PN sequence & Gold sequence: $Q=127$ \\
\hline Channel Coding & RSC Code \\
\hline Code Memory Length & $\nu=6$ \\
\hline Code Rate & $R_{c}=0.5$ \\
\hline Modulation & DQPSK \\
\hline MSDSD Observation Window Size & $N_{w i n d}=6$ \\
\hline Inner Iterations of DN's Decoder & $I_{\text {inner }}^{d}=2$ \\
\hline Outer Iterations of DN's Decoder & $I_{\text {outer }}^{d}=9$ \\
\hline Relay Position in AF Based SRAN & $\theta=\frac{1}{2}$, \\
\cline { 2 - 3 } & $G_{s r_{i}}=\left(\frac{1.0}{0.357}\right)^{3}, G_{r_{i} d}=\left(\frac{1.0}{0.714}\right)^{3}$ \\
\hline Relay Position in DF Based SRAN & $\theta=1.0$, \\
\cline { 2 - 3 } & $G_{s r_{i}}=G_{r_{i} d}=\left(\frac{1.0}{0.577}\right)^{3}$ \\
\hline Overall Bandwidth efficiency & $\eta=0.8333$ bits/s/Hz \\
\hline
\end{tabular}

TABLE II: SYSTEM PARAMETERS

us now investigate the robustness of the proposed three stage relay-aided-MSDSD-URC-RSC decoder in the terms of its BER performance. We commence by identifying the "turbocliff" SNR with the aid of Extrinsic Information Transfer (EXIT) Charts as detailed in [6]. Both the relevant EXITchart and BER results of our AF/DF based SRAN are shown in Fig 9, when using the system parameters summarized in Table II.

The MSDSD scheme requires the symbols of a decisionblock to be correlated. By contrast, we have to eliminate the fading-induced correlations among symbols for the sake of achieving efficient iterative decoding, which is attained by using an interleaver frame length corresponding to $480 \times 10^{3}$ differentially modulated symbols. The adaptive-window-duration based scheme proposed for the SISO-MSDSD algorithm in [9] is capable of reducing the system's complexity, but all the systems involved in our comparisons, such as the conventional direct transmission based system, and the AF or DF based SRANs are capable of directly adopting it. Hence, regardless whether or not we opt for employing the adaptive-windowduration based scheme, this would not affect the preference order of the different systems. Accordingly, we fixed the observation window size of the SISO-MSDSD algorithm to $N_{\text {wind }}=6$ for all the systems. Furthermore, in order to guarantee the fairness of our comparisons among all the systems considered, the overall transmit power $P_{\text {total }}$ is equally shared between the SN and RN transmitters, as suggested in Section III] which equals to the overall power of the direct transmission based system.

Observe in Fig 9a that an open tunnel exists between the EXIT curves of the two-stage inner relay-aided-MSDSDURC decoder and the outer RSC decoder, when the overall equivalent SNR value 5 reaches $2.12 \mathrm{~dB}$. Furthermore, the associated Monte-Carlo simulation based decoding trajectory relying on a frame length of $L=960 \times 10^{3}$ bits closely matches the EXIT curves. Correspondingly, an infinitesimally low BER is expected beyond the SNR $=2.12 \mathrm{~dB}$ point. This is further evidenced in Fig $9 \mathrm{~b}$. The capacity of the proposed DF based SRAN is also characterized in Fig $9 \mathrm{~b}$, which can be directly attained from Fig 5 In our case, the corresponding bandwidth efficiency is $\eta \approx R_{c} \times \log _{2} M_{c} \times \frac{T_{b}-1}{T_{b}}=0.8333$ $\mathrm{bit} / \mathrm{s} / \mathrm{Hz}$. Hence, the proposed transceiver architecture of Fig.7 attains a performance within $2.6 \mathrm{~dB}$ from the capacity of the DF based SRAN. This $2.6 \mathrm{~dB}$ discrepancy may be further reduced for example with the aid of an irregular outer code, as detailed for example in [27].

To elaborate a little further, observe in Fig $9 b$ that an approximately $3.9 \mathrm{~dB}$ power reduction is achieved by the proposed DF scheme in comparison to the classic direct transmission regime. By contrast, its corresponding AF based counterpart attains a more modest power reduction of about $0.5 \mathrm{~dB}$, which is attained at a lower complexity than that of the DF arrangement.

\section{CONCLUSiOnS}

In this contribution, we suppress the successive relaying induced interferences with the aid of the specifically arranged DS-CDMA technique. Then, based on the capacity analysis of Section [II and the simulation results of Section [V] we may reasonably conclude that instead of invoking the distributed turbo coding regime of [25], our proposed transceiver specifically designed for the DF based SRAN allowed us to halve the system complexity. The SNR discrepancy with respect to the associated DF based SRAN's noncoherent DCMC capacity was as low as $2.6 \mathrm{~dB}$. Furthermore, a power reduction of about $3.4 \mathrm{~dB}$ and $3.9 \mathrm{~dB}$ was attained with respect to its $\mathrm{AF}$ and to its direct transmission based counterparts, respectively.

\section{REFERENCES}

[1] G. J. Foschini and M. J. Gans, "On Limits of Wireless Communications in a Fading Environment when Using Multiple Antennas," Wireless Personal Communications, vol. 6, pp. 311-335, Mar 1998.

[2] E. C. Van der Meulen, "Three-Terminal Communication Channels," Advanced Applied Probability, vol. 3, pp. 120-154, 1971.

[3] J. N. Laneman, D. N. C. Tse, and G. W. Wornell, "Cooperative Diversity in Wireless Networks: Efficient Protocols and Outage Behavior," IEEE Transactions on Information Theory, vol. 50, pp. 3062-3080, Dec 2004.

\footnotetext{
${ }^{5}$ Here the terminology of 'equivalent SNR' is defined as the radio of the transmit power with respect to the receiver's noise, which is measured at physically different points.
} 
[4] B. Rankov and A. Wittneben, "Spectral Efficient Protocols for HalfDuplex Fading Relay Channels," IEEE Journal on Selected Areas in Communications, vol. 25, pp. 379-389, February 2007.

[5] Y. Fan, C. Wang, J. Thompson, H. V. Poor, "Recovering Multiplexing Loss through Successive Relaying Using Repetition Coding," IEEE Transactions on Communications, vol. 6, pp. 4484-4493, December 2007.

[6] L. Hanzo, O. R. Alamri, M. El-Hajjar and N. Wu, "Near-Capacity Multi-Functional MIMO Systems: Sphere-Packing, Iterative Detection and Cooperation," John Wiley, May 2009.

[7] C. Luo, Y. Gong, and F. Zheng, "Full Interference Cancellation for TwoPath Relay Cooperative Networks," IEEE Transactions on Vehicular Technology, vol. 60, pp. 343-347, Jan 2011.

[8] L. Lampe, R. Schober, V. Pauli, and C. Windpassinger, "MultipleSymbol Differential Sphere Decoding," IEEE Transactions on Communications, vol. 12, pp. 1981-1985, Dec 2005.

[9] V. Pauli, L. Lampe, and R. Schober, “"'Turbo DPSK” Using Soft Multiple-Symbol Differential Sphere Decoding," IEEE Transactions on Information Theory, vol. 52, pp. 1385-1398, April 2006.

[10] T. S. Rappaport, "Wireless Communications: Principles and Practice," Prentice-Hall, Upper Saddle River, NJ, 2002.

[11] Y. B. Liang and V. V. Veeravalli, "Capacity of Noncoherent TimeSelective Rayleigh-Fading Channels," IEEE Transactions on Information Theory, vol. 50, pp. 3095-3110, Dec 2004.

[12] L. Wang, L. K. Kong, S. X. Ng and L. Hanzo, "Code-Rate-Optimized Differentially Modulated Near-Capacity Cooperation," IEEE Transactions on Communications, vol. 59, pp. 1-11, June 2011

[13] L. K. Kong, S. X. Ng, R. G. Maunder, and L. Hanzo, "Near-Capacity Cooperative Space-Time Coding Employing Irregular Design and Successive Relaying," IEEE Transactions on Communications, vol. 58, pp. 2232-2241, August 2010.

[14] W. Fang, L.-L. Yang and L. Hanzo, "Transmitter-Preprocessing-Assisted Cooperative Downlink Transmission in DS-CDMA Systems Experiencing Propagation Path loss and Nakagami-m Fading," IEEE Transactions on Vehicular Technology, vol. 58, pp. 4182-4192, Oct 2009.

[15] L. Hanzo, L-L. Yang, E-L. Kuan, and K. Yen, Single and Multi-Carrier DS-CDMA : Multi-User Detection, Space-Time Spreading, Synchronisation and Standards. John Wiley \& Sons, 1st ed., 2005.

[16] J. G. Proakis, Digital Communications. Mc-Graw Hill, 3rd ed., 1995.

[17] M. Pursley, "Performance evaluation for phase-coded SSMA communication - part I : System analysis," IEEE Transactions on Communications, vol. 25, pp. 795-799, Aug 1977.

[18] T. Himsoon, W. Su, and K. J. R. Liu, "Differential Transmission for Amplify-and-Forward Cooperative Communications," IEEE Signal Processing letters, vol. 12, pp. 597-600, September 2005.

[19] L. Wang and L. Hanzo, "The Amplify-and-Forward Cooperative Uplink Using Multiple-Symbol Differential Sphere-Detection," IEEE Signal Processing Letters, vol. 16, pp. 913-916, October 2009.

[20] Y. W. Ding, J. K. Zhang and K. M. Wong, "Ergodic Channel Capacity for the Amplify-and-Forward Half-Duplex Cooperative Systems," IEEE Transactions on Information Theory, vol. 55, pp. 713-730, February 2009.

[21] R. Schober and L. Lampe, "Noncoherent Receivers for Differential Space-Time Modulation," IEEE Transactions on Communications, vol. 50, pp. 768-777, May 2002.

[22] A. Hst-Madsen and J. Zhang, "Capacity Bounds and Power Allocation for Wireless Relay Channels," IEEE Transactions on Information Theory, vol. 51, pp. 2020-2040, June 2005.

[23] T. M. Cover and J. A. Thomas, "Elements of Information Theory," John Wiley Sons, 2006.

[24] R.-R. Chen, R. Koetter, U. Madhow and D. Agrawal, "Joint Noncoherent Demodulation and Decoding for the Block Fading Channel: A practical Framework for Approaching Shannon Capacity," IEEE Transactions on Communications, vol. 10, pp. 1676-1689, October 2003.

[25] B. Zhao and M. C. Valenti, "Distributed Turbo Coded Diversity for Relay Channel," Electronics Letters, vol. 39, pp. 786-787, May 2003.

[26] Y. K. Song, and D. Kim, "Convergence and Performance of Distributed Power Control Algorithm for Cooperative Relaying in Cellular Uplink Network," IEEE Transactions on Vehicular Technology, vol. 59, pp. 4645-4651, Nov 2010.

[27] L. Hanzo, Y. Akhtman, M. Jiang and L. Wang, "MIMO-OFDM for LTE, WIFI and WIMAX: Coherent versus Non-Coherent and Cooperative Turbo-Transceivers," John Wiley, 2010.

[28] L. Li and L. Hanzo, "Multiple-Symbol Differential Sphere Detection Aided Successive Relaying in the Cooperative DS-CDMA Uplink," Wireless Communications and Networking Conference (WCNC), March 2011.

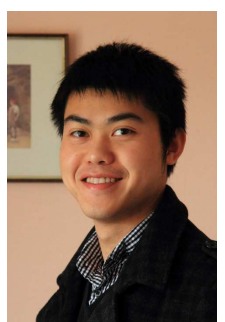

$\mathbf{L i} \mathbf{L i}$ received the B.Eng. degree in information engineering from the University of Electronic Science and Technology of China (UESTC), Chengdu, China, in 2006 and the M.Sc. degree with distinction in wireless communications from the University of Southampton, Southampton, U.K., in 2009. He is currently working towards the Ph.D. degree in the Communications Group, School of Electronics and Computer Science, University of Southampton, Southampton, U.K., and participating in the European Union Concerto project. His research interests include channel coding, iterative detection, non-coherent transmission technologies, cooperative communications and network coding.

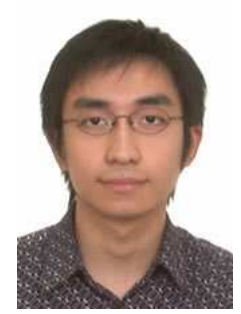

Li Wang (S'09-M'10) was born in Chengdu, China, in 1982. He received his BEng degree in Information Engineering from Chengdu University of Technology (CDUT), Chengdu, China, in 2005 and his MSc degree with distinction in Radio Frequency Communication Systems from the University of Southampton, UK, in 2006. Between October 2006 and January 2010 he was pursuing his $\mathrm{PhD}$ degree in the Communications Group, School of Electronics and Computer Science, University of Southampton, and meanwhile he participated in the Delivery Efficiency Core Research Programme of the Virtual Centre of Excellence in Mobile and Personal Communications (Mobile VCE). Upon completion of his $\mathrm{PhD}$ in January 2010 he conducted research as a Senior Research Fellow in the School of Electronics and Computer Science at the University of Southampton. During this period he was involved in Project \#7 of the IndianUK Advanced Technology Centre (IU-ATC): advanced air interface technique for MIMO-OFDM and cooperative communications. In March 2012 he joined the R\&D center of Huawei Technologies in Stockholm, Sweden, working as Senior Engineer of Baseband Algorithm Architecture. He has published over 30 research papers in IEEE/IET journals and conferences, and he also coauthored one John Wiley/IEEE Press book. He has broad research interests in the field of wireless communications, including PHY layer modeling, link adaptation, cross-layer system design, multi-carrier transmission, MIMO techniques, CoMP, channel coding, multi-user detection, non-coherent transmission techniques, advanced iterative receiver design and adaptive filter.

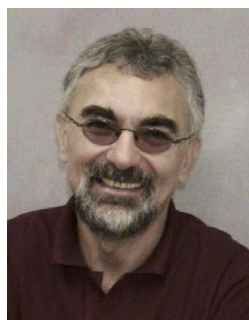

Lajos Hanzo FREng, FIEEE, FIET, Fellow of EURASIP, DSc received his degree in electronics in 1976 and his doctorate in 1983. In 2009 he was awarded the honorary doctorate "Doctor Honoris Causa" by the Technical University of Budapest. During his 35-year career in telecommunications he has held various research and academic posts in Hungary, Germany and the UK. Since 1986 he has been with the School of Electronics and Computer Science, University of Southampton, UK, where he holds the chair in telecommunications. He has successfully supervised $80 \mathrm{PhD}$ students, co-authored 20 John Wiley/IEEE Press books on mobile radio communications totalling in excess of 10000 pages, published 1300 research entries at IEEE Xplore, acted both as TPC and General Chair of IEEE conferences, presented keynote lectures and has been awarded a number of distinctions. Currently he is directing a 100-strong academic research team, working on a range of research projects in the field of wireless multimedia communications sponsored by industry, the Engineering and Physical Sciences Research Council (EPSRC) UK, the European IST Programme and the Mobile Virtual Centre of Excellence (VCE), UK. He is an enthusiastic supporter of industrial and academic liaison and he offers a range of industrial courses. He is also a Governor of the IEEE VTS. During 2008 - 2012 he was the Editor-in-Chief of the IEEE Press and a Chaired Professor also at Tsinghua University, Beijing. His research is funded by the European Research Council's Senior Research Fellow Grant. For further information on research in progress and associated publications please refer to http://www-mobile.ecs.soton.ac.uk 